\title{
Adrenal suppression with chronic dosing of fluticasone propionate compared with budesonide in adult asthmatic patients
}

\author{
D J Clark, B J Lipworth
}

\begin{abstract}
Background - In a previous single dosing comparison between fluticasone propionate and budesonide differences in cortisol levels measured at 08.00 hours were observed at doses in excess of $1000 \mu \mathrm{g}$. The aim of this study was to compare the adrenal suppression caused by chronic twice daily dosing with inhaled fluticasone propionate (FP) and budesonide (B) given on a microgram equivalent basis by metered dose inhaler to asthmatic patients.

Methods - Twelve stable asthmatic patients of mean age 29.7 years with forced expiratory volume in one second $\left(F_{E V}\right)$ $\mathbf{8 9 . 0} \%$ predicted and mid forced expiratory flow $\left(\mathrm{FEF}_{25-75}\right) \quad \mathbf{5 8 . 9 \%}$ predicted, on 400 $\mu \mathrm{g} / \mathrm{day}$ or less of inhaled corticosteroid, were studied in a double blind, placebo controlled, crossover design comparing inhaled budesonide and fluticasone propionate in doses of $250 \mu \mathrm{g}, 500 \mu \mathrm{g}$, and $1000 \mu \mathrm{g}$ twice daily. Each dose was given at 08.00 hours and 22.00 hours for four days by metered dose inhaler with mouth rinsing. Measurements were made of overnight urinary cortisol excretion and plasma cortisol levels at 08.00 hours, 10 hours after the eighth dose.
\end{abstract}

Results - The plasma cortisol levels (nmol/ 1) at 08.00 hours showed that fluticasone propionate produced lower cortisol levels than budesonide at all three dose levels: F500 333.8, B500 $415.2(95 \%$ CI 28.9 to 134.0); F1000 308.3, B1000 380.3 (95\% CI 10.5 to 133.5); F2000 207.3, B2000 318.5 (95\% CI 5.8 to 216.7); placebo 399.9. Fluticasone produced greater effects than budesonide on the overnight urinary cortisol/creatinine ratio $(\mathrm{nmol} / \mathrm{mmol})$ at all three dose levels: F500 3.12, B500 5.55 (95\% CI 0.16 to 3.79); F1000 2.54, B1000 6.12 (95\% CI 1.25 to 5.91); F2000 2.07, B2000 6.09 (95\% CI 0.88 to 7.18$)$; placebo 5.23 .

Conclusions - With repeated dosing across a dose range of $250-1000 \mu \mathrm{g}$ twice daily, fluticasone propionate produced significantly greater adrenal suppression than budesonide for both plasma and urinary cortisol. It was therefore possible to demonstrate differences between fluticasone and budesonide at lower doses with chronic dosing from those previously found with single dosing when given on a microgram equivalent basis in asthmatic patients. Factors contributing to the systemic adverse activity profile of fluticasone comprise enhanced receptor potency, prolonged receptor residency time, greater tissue retention, and a longer elimination half life.

(Thorax 1997;52:55-58)

Keywords: adrenal suppression, chronic dosing, inhaled corticosteroids, asthma, fluticasone propionate, budesonide.

Inhaled corticosteroids have a well established role as first line preventive anti-inflammatory therapy in the treatment of asthma. ${ }^{1}$ Increased use of inhaled corticosteroids has focused greater attention on their systemic side effect profiles. Of these systemic effects, adrenal suppression is the most sensitive accessible marker currently available. In a previous dose ranging study we have revealed significant differences between inhaled fluticasone propionate and budesonide in the degree of adrenal suppression induced when given as single doses on a microgram equivalent basis in asthmatic patients. $^{2}$ These differences between single doses of fluticasone and budesonide can be explained by the pharmacological properties of fluticasone which has both greater glucocorticoid receptor potency ${ }^{3}$ and receptor residency time ${ }^{4}$ than budesonide. However, of greater clinical relevance is the degree of adrenal suppression seen with repeated twice daily dosing as this more accurately reflects what may be expected in clinical practice. In theory, with chronic dosing the longer plasma elimination half life for inhaled fluticasone of 14.4 hours ${ }^{5}$ compared with 2.3 hours for inhaled budesonide $^{6}$ and the greater lipophilicity of fluticasone ${ }^{7}$ will result in greater plasma and tissue retention. This, in conjunction with increased steroid potency and affinity for fluticasone, would be expected to accentuate the differences found between fluticasone and budesonide from the level of suppression already seen with single dosing.

Indeed, in normal subjects a significant increase occurs in adrenal suppression between single and repeated dosing with fluticasone, in keeping with these pharmacological properties. ${ }^{89}$ The aim of this study was to investigate the suppressive effects of fluticasone propionate and budesonide on adrenal activity across a clinically relevant dose range in asthmatic patients.

\section{Methods}

PATIENTS

Twelve asthmatic patients (six women) of mean (SE) age 29.7 (2.4) years completed the study. 
The mean forced expiratory volume in one second $\left(\mathrm{FEV}_{1}\right)$ was $3.07(0.07) 1(89.0(3.71) \%$ of predicted) and mid forced expiratory flow $\left(\mathrm{FEF}_{25-75}\right)$ was $2.49(0.19) \mathrm{l} / \mathrm{s}(58.9(5.1) \%$ of predicted). Haematological and biochemical parameters were normal before entry into the study. All gave written informed consent and approval for the study was given by the Tayside medical ethics committee. All patients had asthma according to the criteria of the American Thoracic Society ${ }^{10}$ and were required to be taking $400 \mu \mathrm{g} /$ day or less of inhaled corticosteroid. The doses of inhaled corticosteroid taken by the 12 subjects were beclomethasone dipropionate $200 \mu \mathrm{g} /$ day (two patients), $300 \mu \mathrm{g} /$ day (one patient), $400 \mu \mathrm{g} /$ day (six patients), budesonide $400 \mu \mathrm{g} /$ day (two patients), and one patient took no inhaled steroids. None had received oral corticosteroids within the preceding three months.

PROTOCOL

A double blind, placebo controlled, randomised crossover design was used with twice daily dosing over four days at each dose level. Subjects attended an initial screening visit where $\mathrm{FEV}_{1}$ and $\mathrm{FEF}_{25-75}$ were measured using a Vitalograph Compact spirometer (Vitalograph Ltd, Buckingham, UK), having withheld inhaled bronchodilators for eight hours. $\mathrm{FEV}_{1}$ was checked on each of the study visits and measurements were only made if it was within $15 \%$ of the baseline value. Inhaler technique was assessed using an aerosol inhalation monitor (Vitalograph Ltd) and detailed instructions in correct usage were given at each visit. Subjects were then randomised to receive fluticasone propionate (Flixotide metered dose inhaler (MDI), $250 \mu \mathrm{g}$ per actuation, Allen and Hanburys, Uxbridge, Middlesex, UK) in doses of $250 \mu \mathrm{g}, 500 \mu \mathrm{g}$, and $1000 \mu \mathrm{g}$ twice daily or budesonide (Pulmicort MDI, $50 \mu \mathrm{g}$ and $200 \mu \mathrm{g}$ per actuation, Astra Pharmaceuticals, Kings Langley, Hertfordshire, UK) in doses of $250 \mu \mathrm{g}$, $500 \mu \mathrm{g}$, and $1000 \mu \mathrm{g}$ twice daily, each dose being given sequentially for four days. Randomisation was in balanced blocks with six receiving fluticasone first and six budesonide first. Placebo MDI was given for four days at the start of each of the two treatment periods. There was at least a 16 day washout period between the two 12 day drug sequences during which the patients resumed their standard inhaled steroid therapy. The aerosol canisters and plastic actuators were masked and placed in identical boxes in order to blind the treatment with respect to the patient. Each treatment box was dispensed by a different person from the investigator to make the study double blind. Subjects omitted their usual steroid inhaler for the duration of each 12 day sequence and took their bronchodilator inhaler $15 \mathrm{~min}$ utes before the study drug at 08.00 hours and 22.00 hours. Mouth rinsing was performed after each of two puffs of their study inhaler. After seven doses at each dose level, immediately before taking the dose at 22.00 hours, subjects emptied their bladder and collected all their overnight urine for 10 hours until the laboratory visit at 08.00 hours the following morning. No further steroid or bronchodilator inhalers were taken until completion of the laboratory visit.

\section{MEASUREMENTS}

The subjects attended the laboratory at 07.30 hours, 10 hours after taking the eighth dose of study drug at each dose level. A cannula was inserted into an antecubital fossa vein to allow blood sampling and subjects then rested supine for 30 minutes. After the rest period blood samples were taken for measurement of serum cortisol levels. The total volume of the overnight 10 hour urine specimen was measured and aliquots were kept for assay of cortisol and creatinine levels.

ASSAYS

Serum and urinary cortisol levels were measured using a commercial radioimmunoassay kit (Incstar, Wokingham, Berkshire, UK). The coefficient of variability (CV) for analytical imprecision within the assay was $4.5 \%$ and between the assays was $8.1 \%$. Urinary creatinine levels were measured on a Cobas-bio autoanalyser (Roche Products Ltd, Welwyn Garden City, Hertfordshire, UK). The intra-assay CV was $4.55 \%$ and interassay was $0.63 \%$.

\section{STATISTICAL ANALYSIS}

All data were analysed using a Statgraphics software package (STSC Software Group, Rockville, Maryland, USA). The data were analysed by comparing placebo with both drugs at each dose level using analysis of variance, followed by Duncan's multiple range testing to observe multiple comparisons. 95\% confidence intervals (CI) for differences between treatments were calculated where significant differences were detected. A probability value of $\mathrm{p}<0.05$ (two tailed) was considered significant.

\section{Results}

Fluticasone propionate produced greater suppression of both 08.00 hour plasma cortisol and overnight urinary cortisol levels than budesonide (fig 1). This effect was evident even at the lowest dose of $250 \mu \mathrm{g}$ twice daily. Estimated equivalent potency values by $\log$ linear interpolation for percentage suppression of 08.00 hour plasma cortisol levels showed a 3.5:1 ratio from comparison of dose-response curves. In other words, $250 \mu \mathrm{g}$ fluticasone twice daily was equivalent to $875 \mu \mathrm{g}$ budesonide twice daily.

Fluticasone produced significantly lower 08.00 hour plasma cortisol levels $(\mathrm{nmol} / \mathrm{l})$ than budesonide at all three doses ranging from $250 \mu \mathrm{g}$ to $1000 \mu \mathrm{g}$ twice daily. Fluticasone produced significant suppression compared with placebo at all doses, whereas budesonide did not at any dose.

Fluticasone also had a greater effect than budesonide on the overnight urinary cortisol/ creatinine ratio $(\mathrm{nmol} / \mathrm{mmol})$ at all three dose 

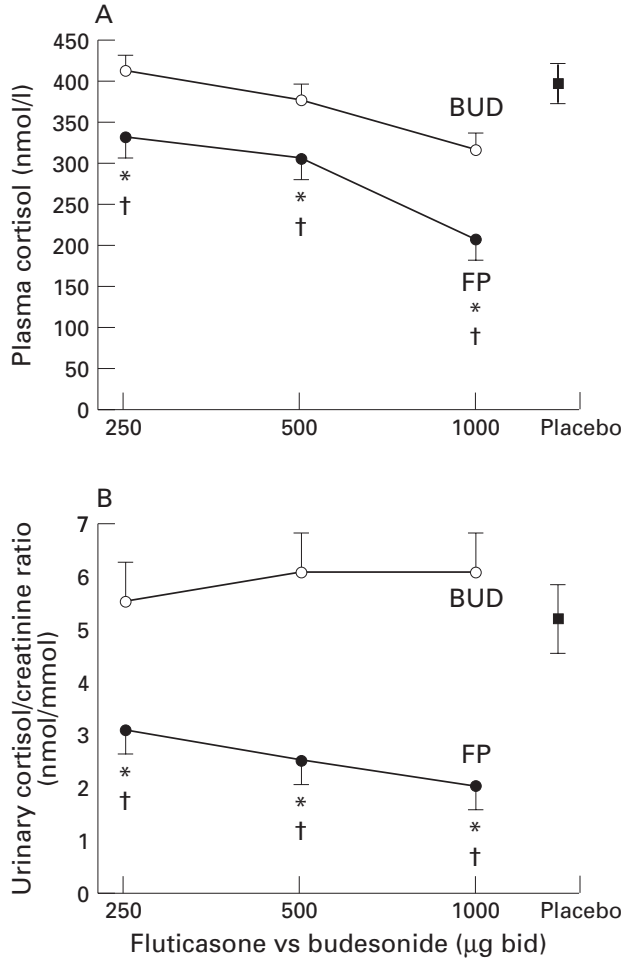

Figure 1 Mean (SE) plasma cortisol concentrations (A) and overnight urinary cortisol/creatinine ratio $(B)$ with repeated twice daily dosing with budesonide (BUD), fluticasone $(F P)$, and placebo. †Significant difference between budesonide and fluticasone at a given dose level; *significant difference between the steroid dose and placebo $(p<0.05)$.
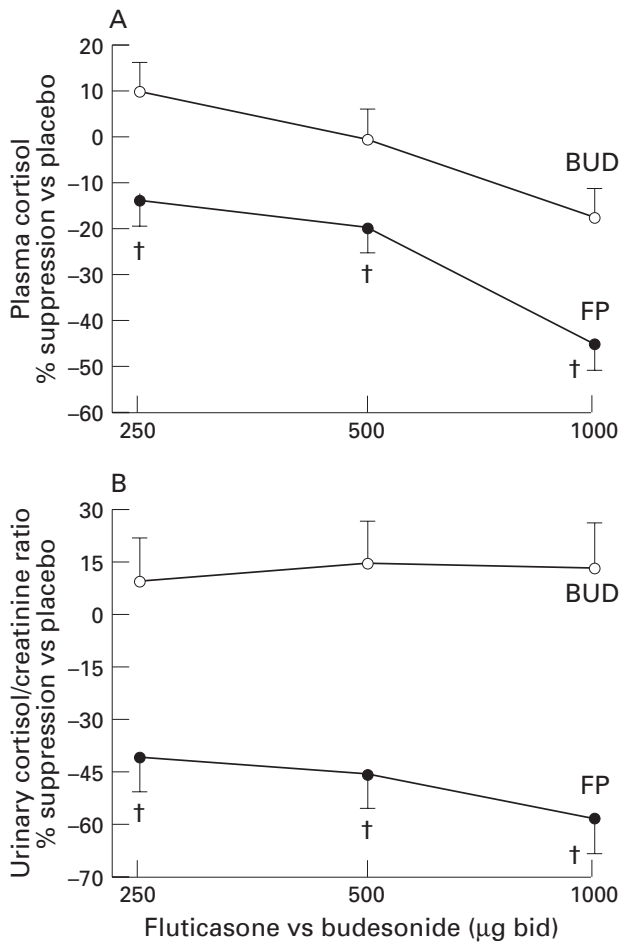

Figure 2 Mean (SE) percentage suppression of plasma cortisol levels compared with placebo $(A)$ and percentage suppression of overnight urinary cortisol/creatinine ratio compared with placebo (B) with repeated twice daily dosing with budesonide (BUD) and fluticasone (FP). †Significant difference between budesonide and fluticasone at a given dose level $(p<0.05)$.

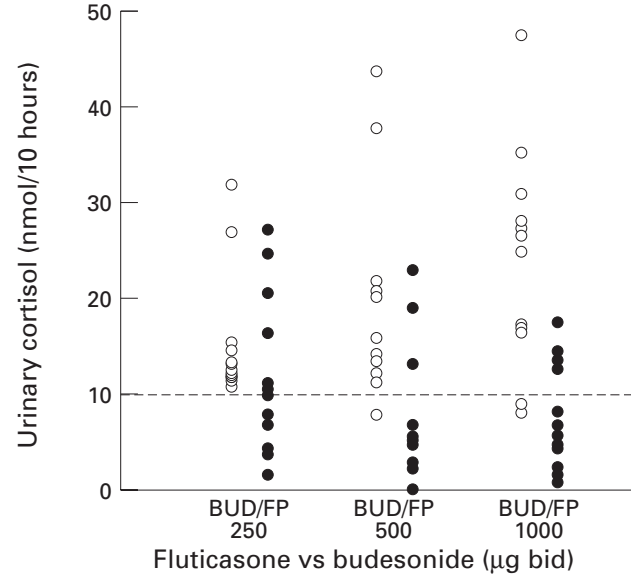

Figure 3 Individual values for overnight urinary cortisol excretion following administration of budesonide (BUD) and fluticasone (FP); 21 of 36 measurements for fluticasone and three of 36 for budesonide $(p<0.005)$ are below $10 \mathrm{nmol} / 10$ hours, shown as an interrupted horizontal line.

levels. It caused significant suppression compared with placebo at all three doses including $250 \mu \mathrm{g}$ twice daily, whereas budesonide caused no significant suppression at even the highest dose of $1000 \mu \mathrm{g}$ twice daily. In terms of percentage suppression versus placebo, fluticasone also produced greater effects at all three dose levels for both plasma cortisol levels and overnight urinary cortisol/creatinine ratio (fig 2).

Individual data for urinary free cortisol levels at all doses are shown in fig 3 to illustrate dispersion and outliers. This showed that 21 of 36 measurements for fluticasone and three of 36 for budesonide were below $10 \mathrm{nmol} / 10$ hours $(\mathrm{p}<0.005)$. There were no significant differences in $\mathrm{FEV}_{1}$ after each four day drug sequence which confirms that variation in lung bioavailability of inhaled steroid or asthma control would be unlikely to affect the cortisol results; fluticasone: baseline 2.93 1, F250 2.99 1, F500 3.02 1, F1000 3.03 1; budesonide: baseline 3.01 l, B250 3.031, B500 2.961, B1000 2.961 .

\section{Discussion}

This study shows that fluticasone propionate causes greater adrenal suppression than budesonide when given on a microgram equivalent basis by metered dose inhaler with repeated twice daily dosing to asthmatic patients across a dose range of $250-1000 \mu \mathrm{g}$ twice daily. A 3.5-fold difference in systemic potency was found between the two drugs which compares with the 5.2-fold difference previously reported by Boorsma et al in normal volunteers for suppression of 08.00 hour cortisol levels. ${ }^{11}$ The differential between fluticasone and budesonide is greater with chronic dosing than that seen previously with single dosing. ${ }^{2}$ This is in keeping with the pharmacological characteristics of these two drugs, with the longer plasma elimination half life, ${ }^{5}$ greater tissue binding, ${ }^{7}$ and prolonged receptor binding ${ }^{4}$ of fluticasone accentuating its greater gluco- 
corticoid potency when given with chronic dosing. In a chronic dosing evaluation of fluticasone $1000 \mu \mathrm{g}$ twice daily against beclomethasone dipropionate $800 \mu \mathrm{g}$ twice daily (Diskhaler) significant adrenal suppression of plasma cortisol levels was observed with fluticasone but not beclomethasone, which was maintained throughout the 12 weeks of the evaluation. ${ }^{12}$ Furthermore, after a further two weeks washout there was still detectable suppression in the fluticasone group, suggesting prolonged body retention. Another factor which may partially explain the observed differences between the two drugs is that the fluticasone MDI produces greater lung deposition than the budesonide MDI, although this is based on in vitro data. ${ }^{13}$ However, since patients often have difficulty using MDIs, putative differences in deposition may become less important in the real life situation.

Comparison between the results of our previous single dosing study ${ }^{2}$ and the present chronic dosing study reveals that this accentuation of the effects of fluticasone results in differences between the two drugs being evident at much lower dose levels with repeated administration. Indeed, with chronic dosing differences in suppression of plasma cortisol levels occurred at a dose of $250 \mu \mathrm{g}$ twice daily, whereas with single dosing a difference in plasma cortisol levels was only demonstrated above $1000 \mu \mathrm{g}$. However, it should be noted that significant differences were observed between single doses of fluticasone $500 \mu \mathrm{g}$ and budesonide $400 \mu \mathrm{g}$ using the more sensitive measure of overnight urinary cortisol levels. In our chronic dosing study the urinary cortisol/ creatinine ratio, which is as sensitive as 24 hour urinary cortisol measurements, ${ }^{14}$ demonstrated significant differences at all doses as would have been predicted from the single dosing results.

The accentuation of the differences between fluticasone and budesonide with chronic compared with single dosing has been demonstrated by Lonnebo et $a l^{9}$ in healthy volunteers. They compared adrenal suppression (as $\mathrm{AUC}_{0-20}$ ) after the first and last doses of fluticasone $1000 \mu \mathrm{g}$ twice daily via Diskhaler with budesonide $800 \mu \mathrm{g}$ twice daily via Turbohaler for seven repeated doses and found a much greater increase in suppression between single and repeated dosing for fluticasone (55\% versus $25 \%$ suppression) than budesonide (34\% versus $26 \%$ ). Boorsma et $a l^{11}$ also found differences between the two drugs when evaluating much lower doses with a chronic dosing regimen. In healthy volunteers $200 \mu \mathrm{g}$ fluticasone MDI twice daily produced $18 \%$ suppression of the 08.00 hours plasma cortisol level compared with placebo, while the same dose of budesonide, also given via an MDI, produced no significant suppression compared with placebo

A comparison of our own data from single and chronic dosing studies also highlights the greater degree of suppression at steady state with fluticasone. Compared with placebo, a single $1000 \mathrm{~g}$ dose produced $6 \%$ suppression of plasma cortisol levels compared with $45 \%$ suppression for chronic dosing with $1000 \mu \mathrm{g}$ twice daily, representing a seven-fold increase. At the $500 \mu \mathrm{g}$ level a single dose caused no suppression compared with placebo, whereas chronic dosing with $500 \mu \mathrm{g}$ twice daily produced a $19 \%$ fall in 08.00 hour cortisol levels. Our results agree with those of Grahnen et al who reported suppression of plasma cortisol (as $\mathrm{AUC}_{0-20}$ ) of $28 \%$ when fluticasone was given as a single dose of $1000 \mu \mathrm{g}$ via a Diskhaler to healthy volunteers compared with $65 \%$ when it was given as $1000 \mu \mathrm{g}$ twice daily for three and a half days.

In conclusion, our results demonstrate that the greater glucocorticoid potency of fluticasone than budesonide translates directly into increased systemic bioactivity as assessed by adrenal suppression using either plasma or urinary cortisol levels. In fact, the complex interplay between factors including receptor potency, receptor residency time, tissue retention, and plasma elimination half life for fluticasone and budesonide results in significantly greater adrenal suppression for fluticasone with repeated administration at doses as low as $250 \mu \mathrm{g}$ twice daily. This highlights the necessity for adequate comparative dose ranging studies of inhaled corticosteroids to characterise fully the dose response relationships of this important class of drugs.

The authors wish to thank Mrs L McFarlane for performing the biochemical assays.

1 British Thoracic Society. Guidelines for management of asthma: a summary. BMF 1993;306:776-82.

2 Clark DJ, Grove A, Cargill RI, Lipworth BJ. Comparative adrenal suppression with inhaled budesonide and fluticasone propionate in adult asthmatic patients. Thora 1996;51:262-6.

3 English AF, Neate MS, Quint DJ, Sareen M. Biological activities of some corticosteroids used in asthma. Am Respir Crit Care Med 1994;149(Suppl):A212.

4 Hogger P, Rohdewald P. Binding kinetics of fluticasone propionate to the human glucocorticoid receptor. Steroids 1994;59:597-602.

5 Thorsson L, Dahlstrom K, Edsbacker S, Callen E, Poulson G, Wirren G. Pharmacokinetics and systemic effects of inhaled fluticasone propionate in healthy subjects. $B r \mathcal{F}$ Clin Pharmacol 1997 (in press)

6 Thorrson L, Edsbacker S, Conradson TB. Lung deposition of budesonide from Turbuhaler is twice that from presof budesonide from Turbuhaler is twice that from pres-
surised metered-dose inhaler. Eur Respir f 1994;7:183944 .

7 Wurthwein G, Rehder S, Rohdewald P. Lipophilicity and receptor affinity of glucocorticoids. Pharm Ztg Wiss 1992 receptor

8 Grahnen A, Eckernas SA, Brundin RM, Ling-Andersson A. An assessment of the systemic activity of single doses of inhaled fluticasone propionate in healthy volunteers. Br F Clin Pharmacol 1994;38:521-5.

9 Lonnebo A, Grahnen A, Jansson B, Brundin RM, LingAndersson A, Eckernas SA. An assessment of the systemic effects of single and repeated doses of inhaled fluticason propionate and inhaled budesonide in normal volunteers. Eur f Clin Pharmacol 1996;49:459-63.

10 American Thoracic Society. Standards for the diagnosis and care of patients with chronic obstructive pulmonary disease (COPD) and asthma. Am Rev Respir Dis 1987;136:225-43

11 Boorsma M, Andersson N, Larsson P, Ullman A. Assessment of the relative systemic potency of inhaled fluticasone and budesonide. Eur Respir ₹ 1996;9:1427-32.

12 Boe J, Bakke P, Rodolen T, Skovlund E, Gulsvik A. Highdose inhaled steroids in asthmatics: moderate efficacy gain
and suppression of the hypothalamic-pituitary adrenal (HPA) axis. Eur Respir F 1994;7:2179-84.

13 Olsson B. Aerosol particle generation from dry powder inhalers: can they equal pressurised metered dose inhalers? inhalers: can they equal pressurised me

14 McIntyre DH, Mitchell CA, Bowler SD, Armstrong JG, Wooler JA, Cowley DM. Measuring the systemic effects of inhaled beclomethasone: timed morning urine collections compared with 24 hour specimens. Thorax 1995;50:1280- 